\title{
Are individual and group treatments equally effective in the treatment of depression in adults? A meta-analysis
}

\author{
Pim Cuijpers \\ Annemieke van Straten \\ Lisanne Warmerdam \\ Department of Clinical Psychology and \\ EMGO, VU University of Amsterdam \\ THE NETHERLANDS
}

\begin{abstract}
Background and Objectives: It is well-established that psychological interventions are effective in the treatment of depression. However, it is not yet clear what the optima format is in which psychological treatments should be delivered. Although several studies have examined whether individual and group treatments are equally effective in the treatment of depression, no comprehensive meta-analysis has examined this.

Methods: We searched major bibliographical databased and conducted a meta-analysis of 15 studies in which individual and group therapies were compared directly to each other.

Results: The mean effect size indicating the difference between individual and group therapies in depressive symptomatology at post-test was 0.20 (95\% CI: [0.05 0.35]; $\mathrm{p}<0.01$ ), in favor of individual therapies, with a lower drop-out rate in individual interventions $(\mathrm{OR}=$ 0.56; 95\% CI: [0.37, 0.86]; $\mathrm{p}<0.01)$. At follow-up no significant differences were found.

Conclusions: Although individual therapy seems to be somewhat more effective than group therapy at the short term, it is not clear whether this is relevant from a clinical point of view. Because of the small number of studies and the limited quality, more research is needed to examine whether the difference between individual and group treatment is clinically relevant.
\end{abstract}

Received 20 November 2007

Revised 20 November 2007

Accepted 1 December 2007

\section{Introduction}

It is well-established that psychological interventions are effective in the treatment of depression, and this is true for a broad spectrum of treatments including cognitive behavior therapy ${ }^{1,2}$, behavioral activation treatments $^{3}$, marital therapy ${ }^{4}$, problemsolving treatments ${ }^{5}$, and interpersonal psychotherapy ${ }^{2}$. These interventions are not only effective in adults with depression, but 
also in older adults ${ }^{6}$, in women with postpartum depression, ${ }^{7}$ and in patients with both depression and general medical disorders, including multiple sclerosis ${ }^{8}$, stroke patients ${ }^{9}$, and cancer patients ${ }^{10}$. A large number of studies has also shown that psychological treatments of depression in children and adolescents are effective, but with smaller effect sizes than in adults ${ }^{11}$.

Most research in this area has focused on the individual treatment of depression, although the number of studies examining group interventions is also substantial ${ }^{12}$. Whether individual and group treatments of depression are equally effective, however, has not yet been established. Some studies have found individual treatment to be more effective than group treatment ${ }^{13,14}$, while others did not find this ${ }^{15-17}$. One problem for studies examining the differences between individual and group treatments is that these differences are probably small. Therefore these studies need very large sample sizes to be able to find these small differences.

Meta-analyses have the advantage that they can integrate the results of individual studies. Therefore they can detect small differences better. In the past few years, the differences between individual and group therapies have been examined in meta-analytic research, but without clear outcomes. In one meta-analy$\operatorname{sis}^{12}$, the effects of group interventions for depression were examined. Although one of the analyses in this study focused on the difference between group and individual interventions, it suffered from several limitations. Not all studies in this area were included (9 studies, compared to the 15 we identified, see below), no test of homogeneity of the sample was conducted, and no analysis of subgroups, follow-up measures, or drop-out rates were conducted. However, this study resulted in some indications that individual and group interventions did not differ from each other significantly. One other meta-analysis ${ }^{2}$ focused only on brief interventions, did not include all relevant studies either (8 of the 15 studies we identified), and did not conduct subgroup analyses. This study did find indications that individual therapies are somewhat more effective than group interventions in the treatment of depression.

The question whether individual and group treatments are equally effective is important for several reasons. First, it is important from the perspective of cost-effectiveness. If group interventions are as effective as individual therapies, this could reduce the costs of treatment considerably, because the costs of group therapy are considerably lower than the costs of individual therapy ${ }^{18}$.

The question whether individual and group treatments are equally effective is also important from a clinical and scientific perspective. When these treatments are indeed equally effective, this suggests that the presence of other patients during the treatment does not interfere with the treatment of the individual patient. On the other hand, it may also be possible that the treatments result in comparable effects but through different mechanisms. For example, in individual treatment the relationship between patient and therapist may be stronger, while in group therapy other mechanisms are more important, such as cohesion and social support ${ }^{19}$.

If individual and group treatments are not equally effective, this could suggest that one of the formats is not as good as the other one in helping the patient to learn the techniques that are helpful in reducing depression. Or it could be possible that group processes interfere with the therapeutic process.

But before these questions can be answered we first have to know whether group and individual treatments are indeed equally effective. Because no clear conclusions could 
be drawn from earlier research, and because of the importance of the question whether both formats are equally effective, we decided to conduct a new meta-analysis.

\section{Method}

\section{Identification and selection of studies}

Studies were traced by means of several methods. First, we used a large database of studies on the psychological treatment of depression in general, which has been described in detail elsewhere ${ }^{3,5,6,20}$. This database was developed through a comprehensive literature search (from 1966 to May 2007) in which we examined 5,178 abstracts in Pubmed (1,224 abstracts), Psycinfo (1,336), Embase (1118) and the Cochrane Central Register of Controlled Trials $(1,500)$. We identified these abstracts by combining terms indicative of psychological treatment (psychotherapy, psychological treatment, cognitive therapy, behavior therapy, interpersonal therapy, reminiscence, life review) and depression (both MeSH-terms and text words). For this database, we also collected the primary studies from 22 metaanalyses of psychological treatment of depres$\operatorname{sion}^{21}$. For the current study, we examined the abstracts of these 777 studies, and selected the ones which included both an individual and a group treatment of depression.

Second, we conducted additional searches in computerized literature databases in which we combined search terms indicative of individual and group therapy (individual OR group), in combination with depression (text and key words) and controlled studies (both text and key words; randomized OR randomized OR clinical OR trial OR experimental). For these additional searches we examined a total of 1,137 abstracts from
Embase (168 abstracts), the Cochrane database (124), Pubmed (293), Psychinfo (364), and Digital Dissertations (188).

In addition, we examined the references of earlier meta-analyses ${ }^{2,12}$, and we reviewed the reference lists of retrieved papers. We also contacted the authors of studies that met inclusion criteria and asked whether they knew of any other (published and unpublished) studies in the field.

We included studies in which ${ }^{1}$ the effects of a group treatment ${ }^{2}$ were compared to an individual treatment ${ }^{3}$ in adults ${ }^{4}$ with a depressive disorder or an elevated level of depressive symptomatology 5 , in a randomized trial. No language restrictions were applied.

\section{Quality assessment}

We assessed the validity of included studies using four basic criteria, as suggested in the Cochrane Handbook ${ }^{22}$ : allocation to conditions is done by an independent (third) party; adequacy of random allocation concealment to respondents; blinding of assessors of outcomes; and completeness of follow-up data.

\section{Meta-analysis}

We calculated effect sizes $(d)$ by subtracting (at post-test) the average score of the individual treatment condition $\left(M_{i}\right)$ from the average score of the group treatment condition $\left(M_{g}\right)$ and dividing the result by the average of the standard deviations of the experimental and control group $\left(S D_{i g}\right)$. An effect size of 0.5 thus indicates that the mean of the experimental group is half a standard deviation larger than the mean of the control group. Effect sizes of .56 to 1.2 can be assumed to be large, while effect sizes of 0.33 to 0.55 are moderate, and effect sizes of 0 to 0.32 are small ${ }^{23}$. 
In the calculations of effect sizes we only used those instruments that explicitly measure depression (Table I). If more than one depression measure was used, the mean of the effect sizes was calculated, so that each study (or contrast group) only had one effect size.

To calculate pooled mean effect sizes, we used the computer program Comprehensive Meta-analysis (version 2.2.021), developed for support in meta-analysis. As we expected considerable heterogeneity, we decided to calculate mean effect sizes with the random effects model. In the random effects model it is assumed that the included studies are drawn from 'populations' of studies that differ from each other systematically. In this model, the effect sizes resulting from included studies differ because of the random error within studies, but also because of true variation in effect size from one study to the next.

In our analyses, we have tested whether there are genuine differences underlying the results of the studies (heterogeneity), or whether the variation in findings is compatible with chance alone (homogeneity) ${ }^{24}$. As indicator of homogeneity, we calculated the $Q$-statistic. A significant $Q$ rejects the nullhypothesis of homogeneity and indicates that the variability among the effect sizes is greater than what is likely to have resulted from subject-level sampling error alone. We also calculated the $I^{2}$-statistic which is an indicator of heterogeneity in percentages as well. A value of $0 \%$ indicates no observed heterogeneity, and larger values show increasing heterogeneity, with $25 \%$ as low, $50 \%$ as moderate, and $75 \%$ as high heterogeneity ${ }^{24}$.

Subgroup analyses and metaregression analyses were conducted according to the procedures as implemented in Comprehensive Meta-analysis version 2.2.021. In the subgroup analyses, we used the mixed effects analyses model, in which the studies in the subgroups were pooled with the random effects model; and we used the fixed effects model to test whether the difference between the subgroups was significant.

We also calculated 'Orwin's fail safe $N$ '. This number indicates how many studies with an effect size of zero should be found in order to reduce the effect size found to a smaller value (for example 0.20). A larger $N$ indicates that the effect size found can be further generalized.

Finally, we examined whether the dropout from the interventions differed in the individual interventions compared to the group interventions. Because drop-out is a dichotomous outcome, we calculated the odds ratio (OR) of drop-out from the interventions (in stead of a standardized effect size). Again, we conducted all meta-analyses with the random effects model, using the Comprehensive Meta-analysis (version 2.2.021) computer program, and we calculated the $Q$-statistic and the $I^{2}$-statistic to estimate heterogeneity between study outcomes.

\section{Results}

\section{Description of studies}

Fifteen studies, with a total of 673 participants (276 in the individual treatment conditions, and 397 in the group treatment conditions) met the inclusion criteria and were included in the current study ${ }^{13-17,25-33}$. The selected characteristics of the included studies are described in Table I. In the fifteen studies, nineteen comparisons between group and individual treatments could be made, because in four studies two group treatments could be compared to an individual treatment.

One study ${ }^{28}$ was very similar to another study $^{29}$, albeit not in all details, and although 
one author was involved in both studies, no reference was made in the later study to the earlier study. It was possible that the data from these two papers were based on the same research. We tried to contact the authors of these papers and tried to get an answer to the question whether these studies were the same. Unfortunately, the authors did not respond. Therefore, we conducted the meta-analyses (see below) both with and without the earlier study. But because the results were very similar (see below), we considered them as two separate studies.

In six studies, participants were recruited from the community, while in the other studies participants were recruited from clinical settings (6 studies), or through systematic screening ( 2 studies). One study did not report the recruitment method. Eleven studies were focused on adults, while two studies focused on specific populations (women with postpartum depression, prison inmates, and multiple sclerosis patients). In twelve studies, the participants had to meet diagnostic criteria for depressive disorder as established with a diagnostic interview. The remaining three studies included participants who scored high on a self-rating depression scale. All studies used the BDI as an outcome measure.

In thirteen of the nineteen comparisons, the intervention in the individual and group interventions were equivalent, while in the other six comparisons different types of interventions were used. We considered the individual and group intervention as equivalent, when the type of treatment and treatment manual were the same (cognitivebehavior therapy, counseling, interpersonal therapy), and when the number of sessions and the length of the sessions was the same in the group and individual format. In eleven of the thirteen comparisons in which an equivalent intervention was used, cognitive behavioral interventions were administered.
The number of sessions in the individual and group interventions varied between six and twenty. Eleven studies were conducted in the United States, three in the United Kingdom, and one in Australia.

\section{Quality of included studies}

The quality of studies varied. In none of the studies it was reported that allocation to conditions was conducted by an independent party. Concealment of random allocation to respondents was not possible or not reported in any of the studies, while blinding of assessors was reported in five studies. Drop-out numbers ranged from 0 to $42.6 \%$. In four studies, intention-to-treat analyses were conducted.

Because the number of studies was small and the quality of included studies was low, we have to conclude that a meta-analyses of the available studies can not give a definite answer to the question whether or not individual and group interventions are equally effective in the treatment of depression. We doubted whether we should conduct a metaanalysis or not, given the small number and quality of studies. We decided, however, to conduct a meta-analysis, in order to formulate hypotheses for future research.

\section{Differences between individual and group therapies at post-test}

The mean effect size indicating the difference between individual and group therapies in depressive symptomatology at post-test was 0.20 (95\% CI: [0.05 0.35]; p<0.01). This indicates that individual therapy is significantly more effective than group therapy, although the effect size indicates a small difference. Heterogeneity, as indicated with $I^{2}$ was zero. The results of these meta-analyses are summarized in Table II and Figure 1. 


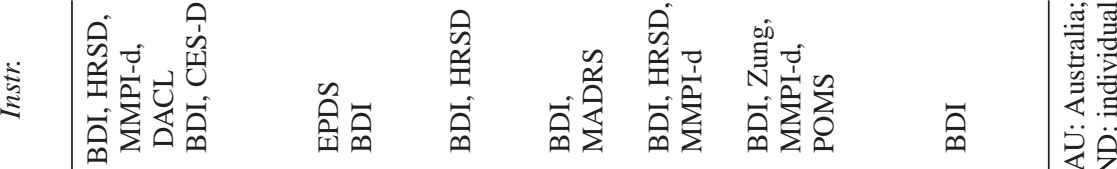

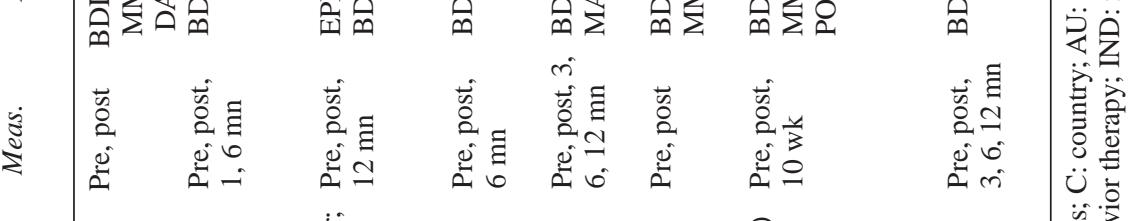

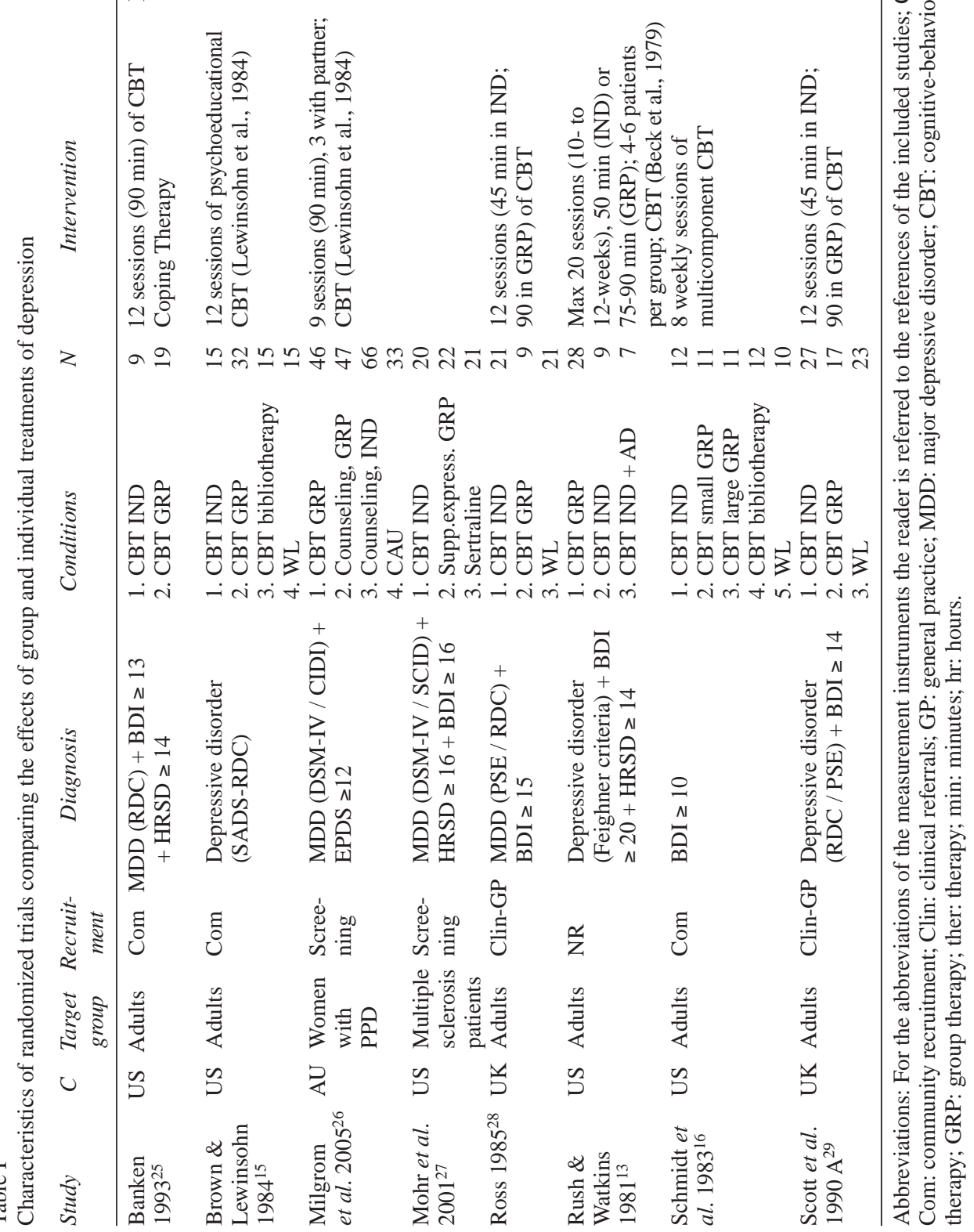




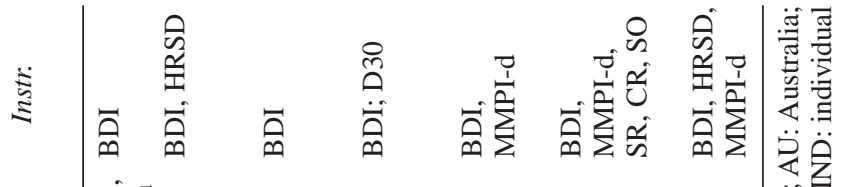

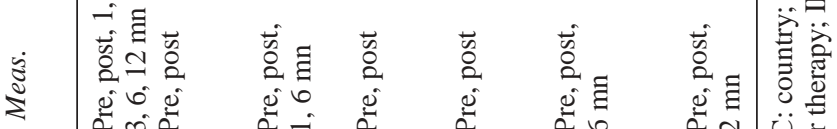

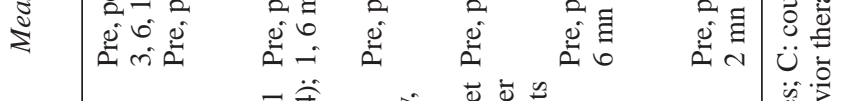

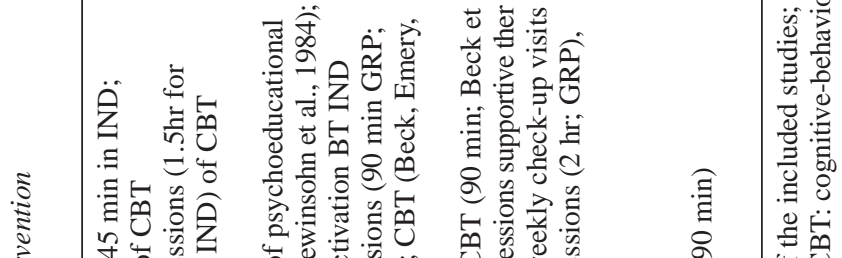
בั

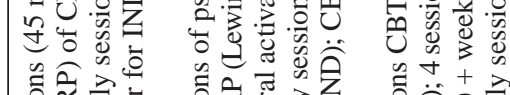

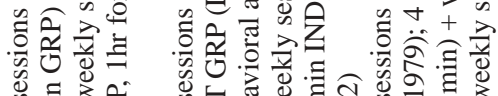

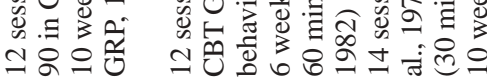

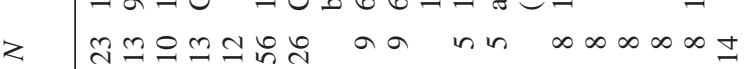

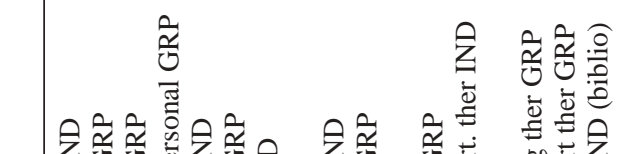

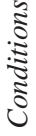

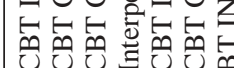

7

원형

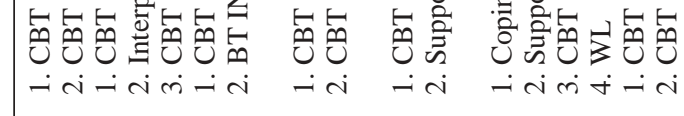

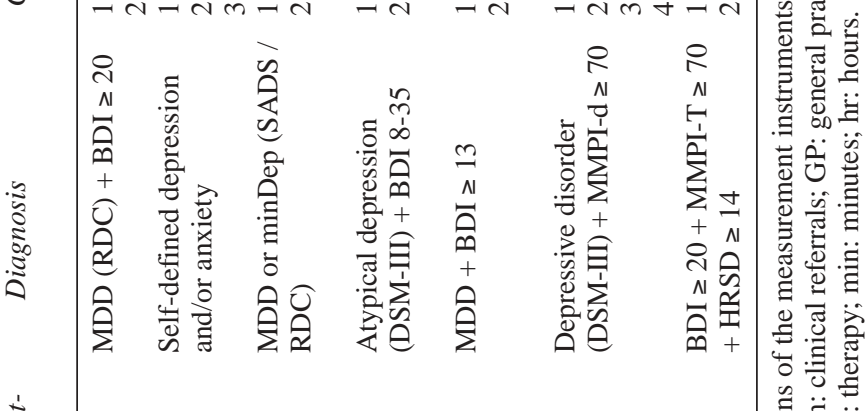

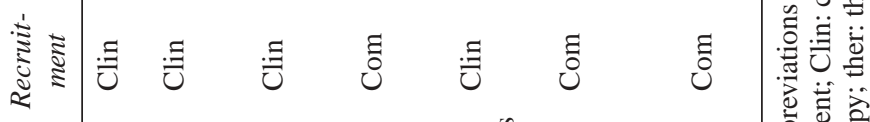

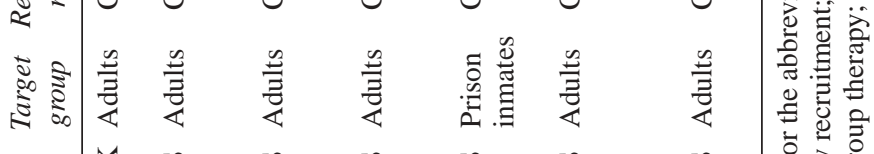

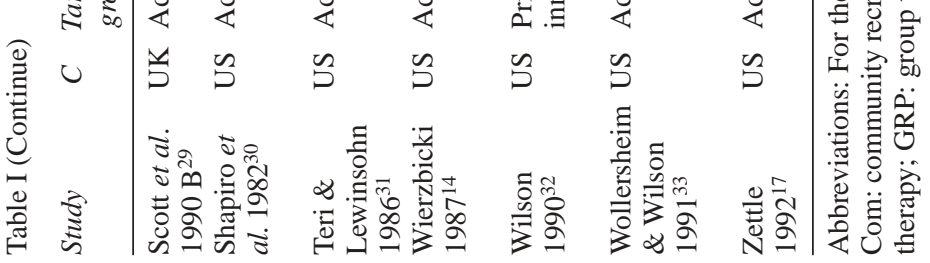


Table II

Meta-analyses of studies comparing the effects of individual psychological treatments to group treatments for depression: Overall results and subgroup analyses

\begin{tabular}{|c|c|c|c|c|c|c|c|}
\hline Study & & $N_{\text {comp }}$ & $95 \% C I$ & $Z$ & $Q^{a)}$ & $I^{2}(\%)$ & $p$ \\
\hline ALL STUDIES & & & & & & & \\
\hline Overall effects & & & & & & & \\
\hline All studies & REM $^{\text {b) }}$ & 19 & 0.200 .050 .35 & $2.60 * *$ & 16.46 & 0 & \\
\hline 1 study excluded ${ }^{c}$ ) & REM & 18 & 0.230 .080 .39 & $2.92 * *$ & 13.83 & 0 & \\
\hline $\mathrm{BDI}{ }^{\mathrm{d})}$ & REM & 19 & 0.280 .120 .44 & $3.38 * *$ & 19.87 & 9.40 & \\
\hline Subgroup analyses & & & & & & & \\
\hline CBT in both formats & Yes & 11 & $0.15-0.070 .38$ & 1.32 & 10.15 & 1.46 & n.s. \\
\hline & No & 8 & 0.240 .040 .44 & $2.31 *$ & 5.99 & 0 & \\
\hline Recruitment & Community & 8 & $0.28-0.020 .58$ & 1.82 & 5.29 & 0 & n.s. \\
\hline & Clinical & 8 & $0.17-0.100 .44$ & 1.25 & 8.58 & 18.43 & \\
\hline & Other & 3 & $0.18-0.100 .45$ & 1.27 & 2.23 & 10.37 & \\
\hline Depressive disorder ${ }^{\mathrm{e}}$ & Yes & 14 & 0.170 .010 .34 & $2.02 *$ & 11.80 & 0 & n.s. \\
\hline & No & 5 & $0.34-0.020 .71$ & $1.84 \mathrm{o}$ & 3.95 & 0 & \\
\hline Specific population $\left.{ }^{f}\right)$ & Yes & 4 & $0.20-0.040 .44$ & 1.62 & 2.70 & 0 & n.s \\
\hline & No & 15 & 0.200 .010 .39 & $2.03 *$ & 13.77 & 0 & \\
\hline Analyses & Compl-only g) & 12 & 0.290 .060 .51 & $2.51 *$ & 9.31 & 0 & \\
\hline & ITT & 5 & $0.08-0.160 .32$ & 0.67 & 4.39 & 8.95 & n.s. \\
\hline Effects at follow-up & & & & & & & \\
\hline 1-3 months follow-up & REM & 7 & $0.05-0.210 .32$ & 0.38 & 7.89 & 23.99 & \\
\hline 6 months follow-up & REM & 7 & $-0.17-0.530 .19$ & -0.92 & 9.89 & 39.31 & \\
\hline STUDIES WITH AN & QUIVALENT IN & NTER & ENTION IN BOTH & RMATS & & & \\
\hline Overall effects & & & & & & & \\
\hline All studies & REM & 13 & $0.18-0.010 .37$ & $1.82 \mathrm{o}$ & 10.31 & 0 & \\
\hline 1 study excluded $^{\mathrm{c}}$ & REM & 12 & 0.230 .030 .42 & $1.22 *$ & 7.83 & 0 & \\
\hline $\mathrm{BDI}{ }^{\mathrm{d})}$ & REM & 13 & 0.270 .050 .48 & $2.38 *$ & 14.69 & 18.30 & \\
\hline Subgroup analyses & & & & & & & \\
\hline CBT in both formats & Yes & 11 & $0.15-0.070 .38$ & 1.32 & 10.15 & 1.46 & n.s. \\
\hline & No & 2 & $0.24-0.120 .59$ & 1.31 & 0.01 & 0 & \\
\hline Recruitment & Community & 7 & $0.30-0.010 .62$ & $1.88 \mathrm{o}$ & 5.05 & 0 & n.s. \\
\hline & Clinical & 4 & $-0.09-0.410 .24$ & -0.51 & 0.78 & 0 & \\
\hline & Other & 2 & $0.31-0.030 .65$ & $1.79 \mathrm{o}$ & 0.79 & 0 & \\
\hline Depressive disorder ${ }^{\mathrm{e}}$ ) & Yes & 9 & $0.10-0.110 .31$ & 0.92 & 5.48 & 0 & n.s. \\
\hline & No & 4 & 0.460 .050 .87 & $2.18 *$ & 2.54 & 0 & \\
\hline Specific population ${ }^{\text {f) }}$ & Yes & 1 & $0.24-0.140 .37$ & 1.25 & 0 & 0 & n.s \\
\hline & No & 12 & $0.15-0.070 .37$ & 1.37 & 10.16 & 0 & \\
\hline Analyses & Compl-only g) & 9 & $0.20-0.060 .46$ & 1.53. & 6.38 & 0 & n.s. \\
\hline & ITT & 4 & $0.11-0.230 .45$ & 0.64 & 4.11 & 27.01 & \\
\hline Effects at follow-up & & & & & & & \\
\hline 1-3 months follow-up & REM & 6 & $-0.04-0.390 .31$ & -0.20 & 6.32 & 20.85 & \\
\hline 6 months follow-up & REM & 4 & $-0.23-0.890 .43$ & -0.69 & $8.62 *$ & 65.22 & \\
\hline
\end{tabular}

$\mathrm{o:} \mathrm{p}<0.1 ; *: \mathrm{p}<0.05 ; * *: \mathrm{p}<0.01$

a) only one of the Q-values was significant;

b) random-effects model;

c) Scott \& Stradling, 1990;

d) effect sizes are exclusively based on the BDI;

e) a depressive disorder as established with a diagnostic interview;

f) women with postpartum depression; prison inmates; and multiple sclerosis patients;

g) completers-only versus intention-to-treat analyses. 
Study name

\begin{tabular}{|c|c|c|c|}
\hline & $\begin{array}{c}\text { Std diff } \\
\text { in means }\end{array}$ & $\begin{array}{l}\text { Lower } \\
\text { limit }\end{array}$ & $\begin{array}{l}\text { Upper } \\
\text { limit }\end{array}$ \\
\hline Banken, 1993 & 0,05 & $-0,92$ & 1,02 \\
\hline Brown, 1984 & $-0,14$ & $-0,81$ & 0,53 \\
\hline Milgrom, $2005 \mathrm{~A}$ & 0,00 & $-0,38$ & 0,38 \\
\hline Milgrom, 2005 B & 0,24 & $-0,14$ & 0,62 \\
\hline Mohr, 2001 & 0,53 & $-0,11$ & 1,17 \\
\hline Ross, 1985 & 0,00 & $-0,56$ & 0,56 \\
\hline Rush, 1981 & 0,65 & $-0,17$ & 1,47 \\
\hline Schmidt, $1983 \mathrm{~A}$ & 0,35 & $-0,47$ & 1,17 \\
\hline Schmidt, 1983 B & 1,06 & 0,19 & 1,93 \\
\hline Scott, $1990 \mathrm{~A}$ & $-0,29$ & $-0,90$ & 0,32 \\
\hline Scott, 1990 B & $-0,10$ & $-0,80$ & 0,60 \\
\hline Shapiro, $1982 \mathrm{~A}$ & 0,13 & $-0,71$ & 0,97 \\
\hline Shapiro, 1982 B & $-0,08$ & $-0,86$ & 0,70 \\
\hline Teri, 1986 & 0,67 & 0,13 & 1,21 \\
\hline Wierzbicki, 1987 & 0,49 & $-0,45$ & 1,43 \\
\hline Wilson, $1990 ?$ & 0,68 & $-0,59$ & 1,95 \\
\hline Wollersheim, 1991 & A 0,20 & $-0,78$ & 1,18 \\
\hline Wollersheim, 1991 & B 0,05 & $-0,93$ & 1,03 \\
\hline \multirow[t]{2}{*}{ Zettle, 1992} & 0,36 & $-0,40$ & 1,12 \\
\hline & 0,20 & 0,05 & 0,35 \\
\hline
\end{tabular}

Statistics for cach study

Z-Value p-Value

0,10

$-0,41$

0,00

1,25

1,63

0,00

1,55

0,83

2,38

$-0,93$

$-0,28$

0,30

$-0,20$

2,41

1,02

1,05

0,40

0,10

0,93

2,60
0,92
0,68
1,00
0,21
0,10
1,00
0,12
0,41
0,02
0,35
0,78
0,76
0,84
0,02
0,31
0,30
0,69
0,92
0,35
0,01

Std diff in means and $95 \%$ CI

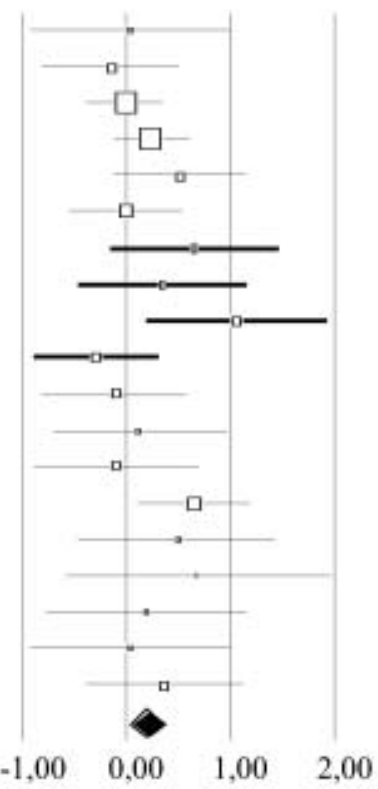

Favours GRP Favours IND

Figure 1. Standardized effect sizes indicating the difference in depressive symptoms between individual and group therapies for depression at post-test.

We calculated Orwin's fail-safe N, which is the number of studies with an effect size of zero that should be found in order to reduce the mean effect size from 0.20 to 0.10 , and this was found to be 19 .

When we excluded the study that was possibly included twice (study 1 by Scott and Stradling $)^{29}$, the results were almost the same $\left(d=0.23\right.$; 95\% CI: [0.08 0.39]; $\mathrm{p}<0.05 ; I^{2}=$ $0)$. Because all the studies used the BDI as an outcome measure, we also examined the difference between individual and group therapies in which the effect size was calculated on the basis of the BDI. Again the results were very similar $(d=0.28 ; 95 \% \mathrm{CI}$ : [0.12 $\left.0.44] ; \mathrm{p}<0.01 ; I^{2}=9.40\right)$. Visual inspection of Figure 1, suggested that one of the comparisons from the study of Schmidt and col- leagues ${ }^{16}$ my be an outlier. When we removed this study from the analyses the results remained almost the same $(d=0.17 ; 95 \% \mathrm{CI}$ : [0.02 0.33]; $\left.\mathrm{Z}=2.22, \mathrm{p}<0.05 ; I^{2}=0\right)$.

We conducted separate analyses for the twelve studies (thirteen comparisons) in which equivalent interventions were examined in the individual and in the group format. The strength of the evidence is clearly stronger when we limit the meta-analysis to the studies in which an equivalent intervention was used in both formats. We decided therefore to repeat all analyses with this subgroup of studies. The results of these analyses are presented in Table II. As can be seen in Table II, these analyses resulted in comparable outcomes, with an overall mean effect size of $0.18(95 \%$ CI: [-0.01 0.37]; $\mathrm{Z}=1.82, \mathrm{p}<0.1 ; I^{2}=0$ ). 


\section{Subgroup analyses}

We conducted some subgroup analyses (Table II). First, we examined whether studies in which cognitive behavioral interventions were used in the individual and the group format differed significantly from studies in which different manuals were used. As can be seen, these two subgroups of studies did not differ significantly from each other $(\mathrm{p}>0.05)$. Then we examined whether studies in which participants were recruited through the community resulted in different mean effect sizes from studies with clinical referrals. Again, no significant difference was found. We also examined whether studies in which participants met diagnostic criteria for a depressive disorder differed from studies in which other inclusion criteria were used, and we compared studies in specific populations (women with postpartum depression, prison inmates, and multiple sclerosis patients) to general adult populations. Furthermore, we examined whether studies in which completers-only analyses were conducted differed significantly from studies in which intention-to-treat analyses were conducted. In none of these analyses were significant differences between subpopulations found. As can be seen from Table II, in most subgroups the difference between individual and group treatments was not significant.

Again, we repeated these analyses for the twelve studies (thirteen comparisons) in which an equivalent interventions were examined in the individual and in the group format (Table II). The results pointed in the same directions as the subgroup analyses with all studies. None of the subgroups differed significantly from other subgroups.

\section{Metaregression analyses}

We conducted a metaregression analysis to examine whether the size of the groups in the group treatments was related to the effect sizes indicating the difference between individual and group treatments. Because we found that individual therapy was somewhat more effective than group treatment, we considered it possible that the difference between group and individual treatment might decrease when the group size in the group treatments was reduced. The group size ranged from 5 to 13 . However, a metaregression analysis did not indicate that the effect size was related to the group size.

We also examined in a metaregression analysis whether the BDI-score at pretest was related to the effect size. A significant result would indicate that the difference of effects in group and individual treatments would be more related to the severity of the depression at pretest. However, these analyses did not indicate either that pretest severity was related to the effect size.

When we limited the analyses to the studies in which equivalent interventions were examined in the individual and in the group format, both the group size and the BDIscore at pretest were not significantly related to the effect size.

\section{Differences in drop-out from interventions}

Next, we examined whether group and individual treatments resulted in different drop-out rates from the interventions. We could calculate these drop-out rates in eleven studies (12 comparison groups). We found that the drop-out rate was significantly lower in individual interventions compared to group interventions ( $\mathrm{OR}=0.56$; 95\% CI: [0.37 0.86]; $\mathrm{p}<0.01)$, with very low heterogeneity $(Q=$ 5.12 , n.s.; $I^{2}=0$ ). This difference remained significant when we removed the study which was possibly included twice $(\mathrm{OR}=0.56 ; 95 \%$ 
CI: [0.36 0.87]; $\mathrm{p}<0.05 ; Q=5.11$, n.s.; $I^{2}=$ 0 ), and when we limited the analyses to the studies in which equivalent interventions were used in the individual and group formats (10 comparisons; $\mathrm{OR}=0.55$; $95 \% \mathrm{CI}$ : [0.32 0.96]; $\mathrm{p}<0.05 ; Q=4.32$, n.s.; $I^{2}=0$ ).

\section{Differences at follow-up}

It was possible to compare the effects of individual and group treatments at one to three months after termination of the interventions in seven comparisons. The mean effect size was not significant $(d=0.05 ; 95 \%$ CI: $\left[\begin{array}{ll}-0.21 & 0.32\end{array}\right)$, while heterogeneity was still low $\left(I^{2}=23.99\right)$. At six months followup, the difference between individual and group treatments was not significant either (seven comparisons, $d=-0.17$; 95\% CI: [-0.53 $0.19])$, with low to moderate heterogeneity $\left(I^{2}=39.31\right)$. No data were available for longer follow-up periods. When we limited the analyses to studies in which equivalent interventions were used in both formats, the effects were comparable again (Table II).

\section{Discussion}

The results of meta-analyses indicate that individual interventions may be more effective than group interventions in the treatment of depression in the short term. At one to six months follow-up, no indications were found that individual and group treatments differed in effectiveness. The difference between individual and group treatments, however, was found to be small $(d=0.20)$.

It is not clear whether such a difference is clinically relevant. Because the costs of group treatments are considerably lower than the costs of individual therapy, it is not clear whether the small difference in effects is worth the costs. Because we also found that the drop-out rate is significantly higher in individual therapy, this may even strengthen reduce the cost-effectiveness of group therapies further.

It has been estimated that the costs of group therapy are about half of the costs of individual therapy ${ }^{18}$. It should be possible to develop a model in which these lower costs were further examined, while including the preferences of patients for each of the two formats, the costs of the interventions, the (possibly) larger effect of individual therapies, and the higher drop-out in group therapies. It should be possible to conduct a modeling study, which would results in a comparison of individual and group therapies in terms of quality of life and economic costs. Such a study is, however, complex and can not be conducted within the context of the current study.

However, the results of our study should be interpreted very cautiously because of the small number of studies, and should at this stage not discourage the development of group treatments, without adequately testing the topic with a more reasonable number of studies.

No indications were found that the difference between group and individual treatments were related to the characteristics of the groups, interventions or studies we examined. Apparently, this difference exists regardless of these characteristics. However, the number of studies in each subgroup is very small and it can not be concluded, therefore, that the subgroup factors are not significant. The fact that we did not find a relationship may be related to the small number of studies in the subgroups.

We also found that the drop-out rate is smaller in individual treatments compared to the drop-out rate in group treatments. It seems that the cohesion between group 
members and the relationship between the patient and the group leader, is not as strong as the personal relation between the patient and his or her individual therapist. Or it may be possible that unpleasant interpersonal events in the group may induce some participants to drop out. This should be the subject for future research, and this finding should also be interpreted very cautiously because of the small number of studies and the low quality of included studies.

This study has several limitations. The first important limitation of this meta-analysis is, as indicated earlier, that we were only able to include a relatively small number of studies, and several of these had very small sample sizes. This small number of studies and participants is especially important, because we did not compare treatments to control conditions, but to each other. In these analyses, small differences should be expected and in order to find small differences, large numbers are needed. Particularly with regard to the longer term, insufficient studies were available to assess the difference between the two types of treatments. A second limitation is that the quality of most included studies was not optimal. Although it is clearly inherent in studies of psychological treatments that it is not possible to conceal to participants to which condition they are assigned (in waiting list control conditions it is not possible at all), many studies did not meet other major quality criteria, such as assignment to conditions by an independent person, and blinding of assessors. Most studies only conducted completers-only analyses instead of intentionto-treat-analyses.

Despite these limitations, our results indicate that individual treatments may be more effective than group treatments. It can easily be an artefact caused by the low quality of the included studies. If this difference is an actual difference, it is not clear what causes this difference. Do individual and group treatments work through different mechanisms, resulting in different effect sizes? Or do they work through the same mechanisms, but are some of these mechanisms limited (in some patients) through the group processes? There is no clear answer to this question yet. It is estimated that psychotherapies work through placebo effects (15\%), extratherapeutic change $(40 \%)$, common factors of psychotherapy $(30 \%)$, and the techniques of the treatment $(15 \%)^{34,35}$. There is no direct reason to assume that placebo effects and extratherapeutic change work differently in group and individual treatments. Most studies in our meta-analysis used the same manuals and protocols in the individual and group formats, and one may expect that the actual techniques in these studies are comparable in both formats. It seems less plausible therefore that the differences in effects of the two formats are caused by the differences in techniques. So, differences between the two formats are probably caused by differences in the common factors. Important common factors include person-centered facilitative conditions (empathy, warmth, congruence) and the therapeutic alliance ${ }^{34}$. In group treatments group cohesion may play a role in realizing effects of an intervention, while individual therapies may strengthen the therapeutic relation between client and therapist more than in group therapies. More research is needed to examine these common factors in both formats.

However, it is also very well possible that individual and group therapies work through comparable mechanisms, but that the group format is limiting these therapeutical mechanisms. For example, it has suggested that there are multiple memories involving the self that compete to be retrieved, and that 
therapies produces changes in the relative activation of positive and negative representations such that the positive ones are assisted to win the retrieval competition ${ }^{36}$. This would mean that therapies may differ from each other considerably, but still realize comparable effects, as long as they succeed in breaking the dominance of negative representations. This would suggest that individual therapies are more successful in this process than group therapies.

This meta-analysis found indications that individual therapies may be more effective than group therapies at the short-term. However, the number of studies, the limited quality of included studies, the uncertainty about the difference at the longer term and about the clinical relevance of this finding, should at this stage not discourage the development of group treatments. More research is needed to examine the exact mechanisms of change in individual and group treatments, before definite conclusions can be drawn.

\section{References}

1. Gloaguen V, Cottraux J, Cucherat M, Blackburn IM. A meta-analysis of the effects of cognitive therapy in depressed patients. J Affect Dis 1998; 49: 59-72.

2. Churchill R, Hunot V, Corney R, Knapp M, McGuire $\mathrm{H}$, Tylee A, et al. A systematic review of controlled trials of the effectiveness and cost-effectiveness of brief psychological treatments for depression. Health Technol Assessm 2001; 5: 35.

3. Cuijpers P, van Straten A, Warmerdam L. Behavioral treatment of depression: A meta-analysis of activity scheduling. Clin Psychol Rev 2007; 27: 318-326.

4. Barbato A, D’Avanzo B. Marital therapy for depression. The Cochrane Database of Systematic Reviews 2006, Issue 2. Art. No.: CD004188.pub2. DOI: 10.1002/14651858.CD00 4188.pub2.
5. Cuijpers P, van Straten A, Warmerdam L. Problem solving therapies for depression: A meta-analysis. Eur Psychiatry $2007 ; 22: 9-15$.

6. Cuijpers P, van Straten A, Smit F. Psychological treatment of late-life depression: A meta-analysis of randomized controlled trials. Int J Geriatr Psychiatry 2006; 21 : 1139-1149.

7. Lumley J, Austin MP, Mitchell C. Intervening to reduce depression after birth: A systematic review of the randomized trials. Int $\mathrm{J}$ Technol Assessm Health Care 2004; 20: 128-144.

8. Mohr DC, Goodkin DE. Treatment of depression in multiple sclerosis: Review and meta-analysis. Clin Psychol Sc Pract 1999; 6: 1-9.

9. Hackett ML, Anderson CS, House AO. Interventions for treating depression after stroke. The Cochrane Database of Systematic Reviews 2004, Issue 2. Art. No.: CD003437.pub2. DOI: 10.1002/14651858.CD003437.pub2.

10. Sheard T, McGuire P. The effect of psychological interventions on anxiety and depression in cancer patients: results of two meta-analyses. Br J Cancer 1999; 80: 1770-1780.

11. Weisz JR, McCarty CA, Valeri SM. Effects of psychotherapy for depression in children and adolescents: a meta-analysis. Psychol Bull 2006; 132: 132-149.

12. McDermut W, Miller IW, Brown RA. The efficacy of group psychotherapy for depression: A meta-analysis and review of the empirical research. Clin Psychol Sc Pract 2001; 8: 98-116.

13. Rush AJ, Watkins JT. Group versus individual cognitive therapy: A pilot study. Cogn Ther Res 1981; 5: 95-103.

14. Wierzbicki M, Bartlett TS. The efficacy of group and individual cognitive therapy for mild depression. Cogn Ther Res 1987; 11: 337-342.

15. Brown RA, Lewinsohn PM. A psychoeducational approach to the treatment of depression: comparison of group, individual and minimal contact procedures. J Consult Clin Psychol 1984; 52: 774-783.

16. Schmidt MM, Miller WR. Amount of therapist contact and outcome in a multidimensional depression treatment program. Acta Psychiatr Scand 1983; 67: 319-332.

17. Zettle RD, Haflich JL, Reynolds RA. Responsivity to cognitive therapy as a function of treatment format and client personality dimensions. J Clin Psychol 1992; 48: 787-797.

18. Vos T, Corry J, Haby MM, Carter R, Andrews G. Cost-effectiveness of CBT and drug interventions for major depression. Austr Nw Zeal J Psychiatry 2005; 39: 683-692. 
19. Yalom ID. The theory and practice for group psychotherapy. Basic Books, New York, NY, 1975.

20. Cuijpers P, van Straten A, Smit F. Psychological treatments of subthreshold depression: A meta-analytic review. Acta Psychiatr Scand 2007; 115: 434441.

21. Cuijpers P, Dekker J. Psychologische behandeling van depressie: een systematisch overzicht van meta-analyses. Ned Tijdschr Geneesk 2005; 149: 1892-1897. [Psychological treatment of depression: A systematic review of meta-analyses. Dutch Journal of Medicine]

22. Higgins JPT, Green S. Cochrane handbook for systematic reviews of interventions 4.2.5 [updated May 2005]. In: The Cochrane Library, Issue 3, 2005. John Wiley \& Sons, Ltd., Chichester, UK.

23. Lipsey MW, Wilson DB. The efficacy of psychological, educational and behavioral treatment. Am Psychol 1993; 48: 11811209.

24. Higgins JP, Thompson SG, Deeks JJ, Altman DG. Measuring inconsistency in meta-analyses. BMJ 2003; 327: 557-560.

25. Banken DM. Group versus individual cognitivebehavioral treatment for depression. Washington State University, Dissertation, 1993.

26. Milgrom J, Negri LM, Gemmill AW, McNeil M, Martin PR. A randomized controlled trial of psychological interventions for postnatal depression. Br J Clin Psychol 2005; 44: 529-542.

27. Mohr DC, Boudewyn AC, Goodkin DE, Bostrom A, Epstein L. Comparative outcomes for individual cognitivebehavior therapy, supportive-expressive group psychotherapy, and sertraline for the treatment of depression in multiple sclerosis. J Consult Clin Psychol 2001; 69: 942-949.

28. Ross M, Scott M. An evaluation of the effectiveness of individual and group cognitive therapy in the treatment of depressed patients in an inner city health centre. J Roy Coll Gen Pract 1985; 35: 239-242.
29. Scott MJ, Stradling SG. Group cognitive therapy for depression produces clinically significant reliable change in community-based settings. Behav Psychoth 1990; 18: 1-19.

30. Shapiro J, Sank LI, Shaffer CS, Donovan DC. Cost effectiveness of individual vs. group cognitive behavior therapy for problems of depression and anxiety in an HMO population. J Clin Psychol 1982; 38: 674-677.

31. Teri L, Lewinsohn PM. Individual and group treatment of unipolar depression: Comparison of treatment outcome and identification of predictors of successful treatment outcome. Behav Ther 1986; 17: 215-228.

32. Wilson GL. Psychotherapy with depressed incarcerated felons: A comparative evaluation of treatments. Psychol Rep 1990; 67: 1027-1041.

33. Wollersheim JP, Wilson GL. Group treatment of unipolar depression: a comparison of coping, supportive, bibliotherapy, and delayed treatment groups. Prof Psychol Res Pract 1991; 22: 496-502.

34. Lambert MJ, Barley DE. Research summary on the therapeutic relationship and psychotherapy outcome. Psychother 2001 ; 38: 357-361.

35. Norcross JC. Psychotherapy relationships that work. New York: Oxford University Press, 2002.

36. Brewin CR. Understanding cognitive behaviour therapy: A retrieval competition account. Behav Res Ther 2006; 44: 765-784.

Address for correspondence:

Pim Cuijpers, Ph.D.

Professor of Clinical Psychology

Department of Clinical Psychology and EMGO

VU University Amsterdam

Van der Boechorststraat 1

1081 BT Amsterdam

The Netherlands

Tel. + 31205988757

Fax. + 31205988758

Email. P.Cuijpers@psy.vu.nl 